\title{
BEAVRS BENCHMARK EVALUATION WITH CASMO5 AND SIMULATE5
}

\author{
Tamer Bahadir \\ Studsvik Scandpower, Inc., \\ 10 Langley Rd, Suite 201, Newton, MA 02459, USA \\ tamer.bahadir@studsvik.com
}

\begin{abstract}
The MIT BEAVRS benchmark problem, which was primarily setup for the verification and validation of high-fidelity tools that have coupled neutron transport, thermal-hydraulics, and fuel isotopic depletion models, has also found extensive usage in the reactor physics community for validating core analysis tools. The primary purpose of this paper is to provide an accurate, comprehensive evaluation of the BEAVRS benchmark with CASMO5 and SIMULATE5 codes. The CMS5 calculated results for low-power physics tests (hot zero power critical boron, control rod worth and isothermal temperature coefficients) and full power operation (boron let-down and flux map reaction rate distributions) are compared to plant measured data provided in the benchmark specification. The CMS5 model, using ENDF/BVII.1 nuclear data library, predicts HZP critical boron concentration for all-rods-out conditions within $10 \mathrm{ppm}$ for Cycle-1, and $25 \mathrm{ppm}$ in Cycle-2; the control rod worth is predicted with a difference of $0.7 \% \pm 3.8 \%$, where the maximum difference is less than $10 \%$. For the core follow calculations at the hot full power condition, the average difference in predicting the critical boron concentration is less than $20 \mathrm{ppm}$. In addition, the radial and nodal reaction rate distributions are predicted with a mean difference of about $1.6 \%$ and $3.8 \%$, respectively. The CMS5 calculations are repeated using the most recent ENDF/B-VIII.0 library. No significant difference is observed in predicting measured plant parameters with different nuclear data libraries. Additionally, the impact of various modeling options, which are typically employed with nodal diffusion codes, on the predictions of important core parameters are presented as part of the benchmark evaluation.
\end{abstract}

KEYWORDS: CASMO5, SIMULATE5, BEAVRS

\section{INTRODUCTION}

Studsvik's in-core fuel management code package CMS/CMS5, which includes lattice physics code CASMO4/5 [1] and 3D nodal code SIMULATE3/5 [2], has been widely used in the industry for LWR reactor analysis for more than 35 years. The initial lattice physics code, CASMO-3, and the 3D nodal code, SIMULATE-3, developed in early 1980s were replaced with the state-of-the-art CASMO5 and SIMULATE5 codes in mid 2000s by taking advantage of today's modern computer resources to implement advanced physics and numerical models, as well as employing new nuclear data libraries. The CMS/CMS5 
code package has been applied to approximately 200 reactors, analyzing more than a thousand cycles. It is proven to be accurate for typical LWR reactors.

The MIT BEAVRS reactor, as described in reference [3], contains information for the initial cycle 1 and the reloaded cycle 2 of a typical 4-loop Westinghouse reactor. The benchmark contains not only the detailed description of the reactor core, its fuel and other essential components, but also provides detailed measured data at hot-zero-power and at-power conditions. The benchmark was setup primarily for the verification and validation of high-fidelity tools that have coupled neutron transport, thermal-hydraulics, and fuel isotopic depletion models $[4,5,6]$. Public availability of such detailed data makes this a valuable tool for validating any in-core fuel management code package. As such, partial results for the BEAVRS benchmark using Studsvik's in-core fuel management code package CMS and CMS5, including CASMO5 and SIMULATE5, have been presented by CMS users at past conferences $[7,8]$. The primary purpose of this paper is to provide an accurate and comprehensive evaluation of the BEAVRS benchmark with CASMO5 and SIMULATE5 codes. Additionally, the impact of various modeling options, which are typically employed with nodal diffusion codes, on the predictions of important core parameters are presented. The modeling options considered include: the number of energy groups in the nodal solution, the use of advanced radial re-homogenization models to correct the cross-sections generated with zero net current boundary condition, the reflector data generation options from lattice physics codes (the use of conventional $1 \mathrm{D}$ vs $2 \mathrm{D}$ transport calculations) and the modeling of asymmetric fuel assemblies.

\section{CASMO5 AND SIMULATE5 OVERVIEW}

CASMO5 is a two-dimensional characteristics-based neutron and gamma transport theory lattice physics code with depletion capability. CASMO5 generates cross sections and discontinuity factors for both square lattice BWR/PWR and hexagonal lattice, VVER, diffusion theory 3-D nodal core analysis. CASMO5 includes many advancements both in physics modeling and capabilities, including an updated neutron data libraries containing more than one thousand unique nuclides and materials, a refined 586 energy-group structure, Characteristics-based Dancoff, and enhanced geometry modeling, extended depletion chains solved with Chebyshev Rational Approximation Method (CRAM), and quadratic gadolinium depletion. Ref. [1] presents the details of some of the basic CASMO5 capabilities and features.

SIMULATE5 is a three-dimensional multi-group analytical nodal diffusion code for both square and hexagonal geometries. The major features of SIMULATE5, specific to PWRs, can be summarized as:

- Any number of energy groups may be used.

- In the axial direction, the heterogeneities caused by the presence of spacers, control rod material zones, enrichment and BA zoning, and staggered assembly heights are treated explicitly.

- In the radial direction, an assembly is divided into $\mathrm{N} \times \mathrm{N}$ 'submeshes' (typically $\mathrm{N}=5$ ). Cross sections are provided for each submesh and the multi-group diffusion (or SP3) equation is solved.

- The shortcomings in the conventional approach of generating radial homogenized cross-sections and discontinuity factors from a 1D fuel/reflector transport calculation have been eliminated either by re-computing the reflector node cross-sections and discontinuity factors in real core geometry by using the submesh calculation model or using location dependent radial reflector submesh cross-sections generated from 2D transport calculations.

- The cross sections are described by a hybrid microscopic-macroscopic model. For the whole node, 17 actinides and more than 30 fission products are tracked. In addition, for each submesh, the five most important actinides are followed.

- The pin fluxes/powers and the detector reaction rates are based on the fine scale solution obtained via the axial homogenization and the radial submesh models.

- The embedded fuel temperature calculation is part of the new detailed thermal/hydraulic module. 
Ref. [2] presents the details of SIMULATE5 features.

\section{BEAVRS BENCHMARK PROBLEM}

The MIT BEAVRS benchmark provides a detailed core and fuel description of the initial two cycles of a 4loop Westinghouse reactor:

Cycle 1 contains 3 different fuel types with respect to U-235 enrichment (1.6\%, 2.4\% and 3.1.\%) and 5 different configurations of PYREX ${ }^{\circledR}$ boron glass BP (Burnable Poison/Absorber pins) loaded.

Cycle 2 contains 129 shuffled assemblies from EOC-1, two different fresh fuel types with respect to U-235 enrichment and three different configurations of PYREX ${ }^{\circledR}$ boron glass BP (Burnable Poison/Absorber pins). For all shuffled fuel from cycle 1, the PYREX ${ }^{\circledR}$ boron glass BP has been withdrawn in cycle 2.

\section{CMS5 BEAVRS CORE MODEL}

The core model of the BEAVRS benchmark is set up with the conventional two-step procedure: using crosssections generated from 2D CASMO5 calculations in 3D SIMULATE5 calculations.

\subsection{CASMO5 Fuel and Reflector Models}

CASMO5 2D single assembly calculations, either in octant, quarter or full assembly geometry, are set up for all axial variation of the fuel within the active length. The variations in the axial regions include fuel with and without Burnable Poison (BP) or control rod as well as guide tubes with different dimensions, generally referred to as "DASHPOT" and "No DASHPOT" regions. The spacers in the active fuel zone are modeled as an additional delta cross-section dependency, generated from CASMO5 branch calculations, and are included in the SIMULATE5 library.

The cross-section library for the top and bottom reflectors are generated from conventional one-dimensional fuel and reflector CASMO5 calculations. The top and bottom reflector geometries account for all material compositions within the first $35 \mathrm{~cm}$ above the top and below the bottom of active fuel. Both the conventional radial reflector model using nodal cross-section data generated from single assembly fuel and reflector CASMO5 calculations and the improved radial reflector model [9], using location dependent submesh radial reflector data generated from multi-assembly CASMO5 calculations, are employed for this benchmark problem. The conventional, homogenized, and the improved, position-dependent (for nine locations), radial reflector segments are generated from a single assembly fuel/reflector transport and a multi-assembly transport calculation respectively and are depicted in Fig. 1.

CASMO5 calculations are performed using two different evaluated nuclear data libraries: the default CASMO5 E7R1 library, which is based on ENDF/B-VII-1 evaluation, and recently released E8R0 CASMO5 library based on the latest ENDF/B-VIII.R0 evaluation [10]. 

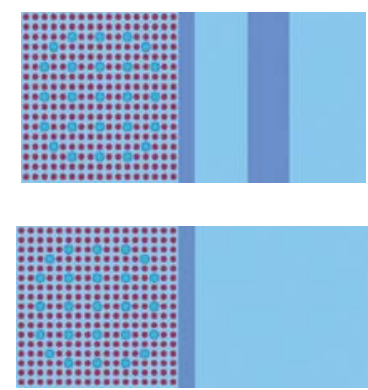

1D, single-assembly, reflector model

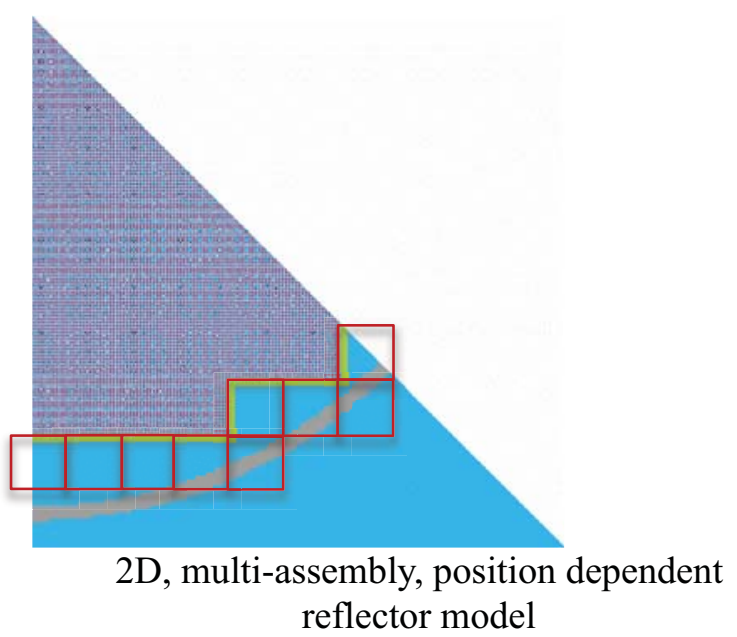

Figure 1. CASMO5 single and multi-assembly geometries for generating radial reflector data.

\subsection{SIMULATE5 Core Model}

The 3D core models are setup with SIMULATE5 using the cross-section libraries generated from CASMO5 transport calculations described in the previous section. Three sets of SIMULATE5 calculations are performed. The simplified SIMULATE5 model uses calculational options typically employed with conventional nodal codes. The default SIMULATE5 model uses all advanced features, which are the default options, of SIMULATE5. Both SIMULATE5 models are run with CASMO data with E7R1 library. In addition, the default SIMULATE5 model evaluations are repeated with the E8R0 library. The major computational options used with each model are compared in Table I.

Table I. Major calculation options of three CMS5 models.

\begin{tabular}{|l|l|l|l|}
\hline Model & $\begin{array}{l}\text { Simplified S5 Model } \\
\text { (SMPL/E7) }\end{array}$ & $\begin{array}{l}\text { Default S5 Model } \\
\text { (DFLT/E7) }\end{array}$ & $\begin{array}{l}\text { Default S5 Model } \\
\text { (DFLT/E8) }\end{array}$ \\
\hline CASMO5 Library & ENDF/B-VII.1 & ENDF/B-VII.1 & ENDF/B-VIII.0 \\
\hline \# energy group & 2 & 4 & 4 \\
\hline $\begin{array}{l}\text { Re-homogenization } \\
\text { Model }\end{array}$ & No & Yes (w/submesh model) & Yes (w/submesh model) \\
\hline Depletion Model & $\begin{array}{l}\text { Macroscopic depletion } \\
\left({ }^{135} \mathrm{I}^{/ 135} \mathrm{Xe},{ }^{149} \mathrm{Pm} /{ }^{149} \mathrm{Sm}\right)\end{array}$ & $\begin{array}{l}\text { Hybrid Macro/Micro Depl. } \\
17 \text { Actinides, 30+ FP }\end{array}$ & $\begin{array}{l}\text { Hybrid Macro/Micro Depl. } \\
17 \text { Actinides, 30+ FP }\end{array}$ \\
\hline Reflector Model & $1 \mathrm{D}$, homogenized, reflector & 2D, submesh, reflector & 2D, submesh, reflector \\
\hline Relative Run Time & 1 & 5.3 & 5.3 \\
\hline
\end{tabular}

The two-group library has the conventional thermal group cut-off at $0.625 \mathrm{eV}$, whereas the four-group library has energy group boundaries at $1 \mathrm{MeV}, 5.53 \mathrm{keV}$, and $0.625 \mathrm{eV}$. All three models have the following common features:

- Each assembly is modelled as 4 nodes/assembly with 24 axial nodes. In addition, the submesh option used with the default model divides each quadrant into 5 by 5 submeshes.

- As-built heavy metal loadings for every assembly loaded in cycles 1 and 2, as provided in the benchmark, are used.

- The detector tubes, which are inserted into the instrument tube location at selected assembly locations, replacing the coolant with the air-filled stainless-steel tubes, are modeled explicitly. 
- The flux map evaluations are performed utilizing the benchmark provided, pre-processed (aligned and calibrated), measured reaction rate data files.

- For the core follow calculations, the operating conditions are taken from the benchmark specification. The core inlet temperature data, available from flux map data files, is used to develop a fit vs. power. Fig. 2 shows the fitted core inlet temperature data. The coolant inlet temperature of $560 \mathrm{~K}$ is assumed for the hot zero power calculations of both cycles.

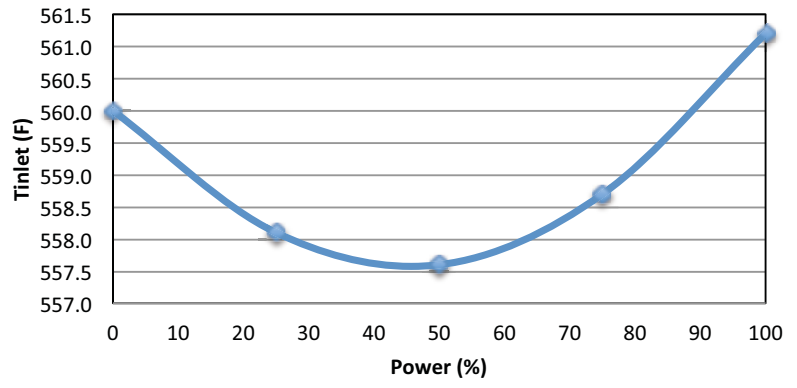

Figure 2. Fitted core inlet temperature data vs. core power.

\subsection{Modeling of Asymmetric Fuel Assemblies}

The initial cycle of the BEAVRS core contains asymmetric fuel assemblies with 6 and 15 BP pins loaded on core edges to flatten the power distribution. Fig. 3 depicts various modeling options that can be used for generating cross-section data for asymmetric fuel assemblies (example below demonstrates the design with $6 \mathrm{BPs})$.

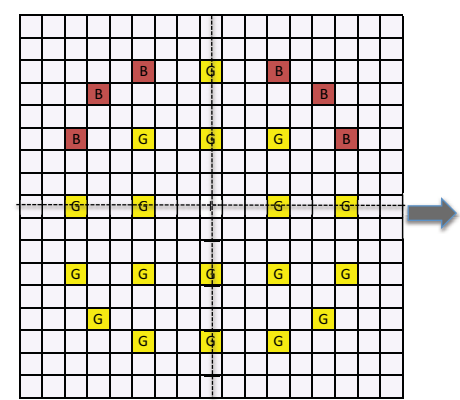

Full Asymmetric Assembly

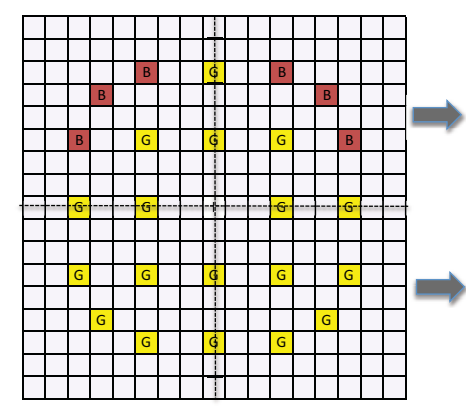

Full Asymmetric Assembly

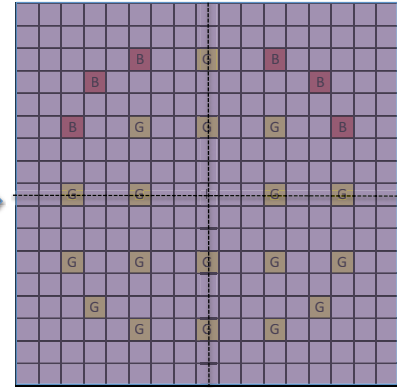

(a) 4 Node/Assm. Model Using Full Assm CASMO Data

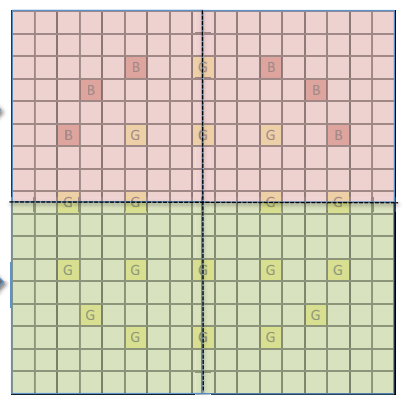

(c) 4 Node/Assm. Model Using Full Assm Data Extracted from Full Assm CASMO data

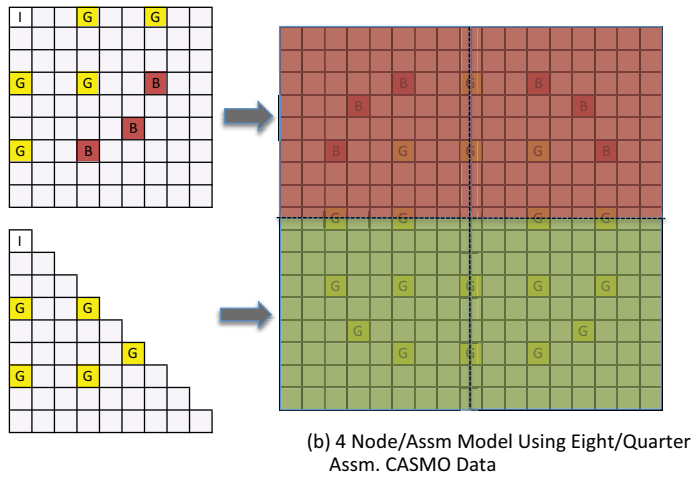

(b) 4 Node/Assm Model Using Eight/Quarter
Assm. CASMO Data

Figure 3. Asymmetric assembly modeling options. 
a. Full assembly model: The nodal cross-section data is generated from full assembly CASMO calculations with periodic boundary conditions and are used in each quadrant of the full assembly in a nodal model.

b. Conventional quarter assembly model: The cross-section data is generated from multiple, either octant or quarter assembly CASMO calculations, one for each unique quadrant, with periodic boundary condition. The asymmetric fuel assembly is then put together in the nodal code where each quadrant uses its own nodal data, including cross-sections/assembly discontinuity factors and pin form factors. This conventional model has been available in CMS since its early inception [11].

c. Advanced quarter assembly model: The quarter assembly nodal data, for each quadrant, is extracted from the solution of a full assembly CASMO calculation, in the linking code level. The advanced model better captures the radial heterogeneity particularly in the pin form factors for those pins close to the internal symmetry axes compared to the conventional model.

The sensitivity of the nodal solution to the modeling of the asymmetric fuel assemblies is examined by evaluating the cycle 1, hot-zero-power (HZP) flux map. Fig. 4 below compares SIMULATE5 predicted detector responses with three different models against the "Tilt Corrected" measured detector responses, which are folded into an octant core geometry, as given in Ref [3]. The large errors at asymmetric fuel assembly locations, highlighted in the measured reaction rate map, and the in and out tilt has been eliminated with the use of quarter assembly data models. The improvement of the advanced quarter model is not obvious from the reaction rate comparisons as the main improvement is in pin powers, for which no measurement is available for comparisons. Accordingly, the advanced quarter assembly model is selected to be used for the rest of this study.

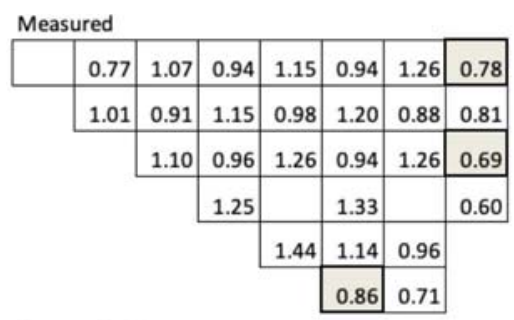

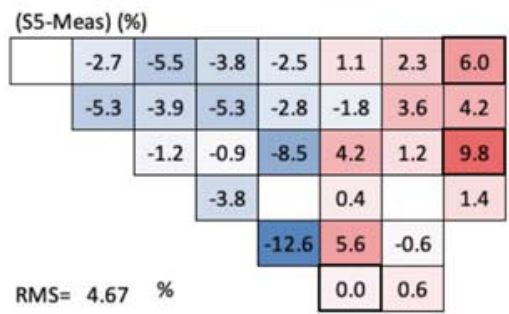

(a) Full Assm Model

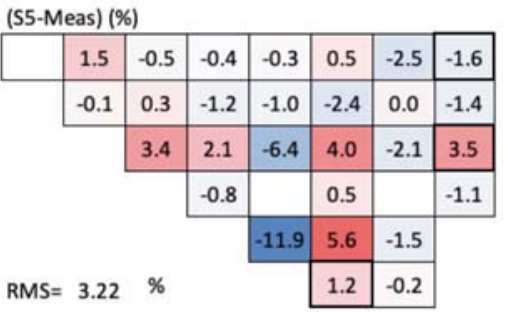

(b) Conventional Quarter Assm Model

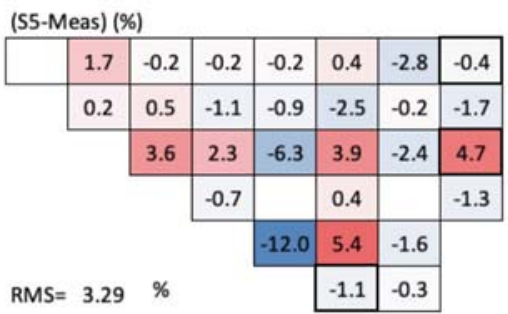

(c) Advanced Quarter Assm Model

Figure 4. Evaluation of cycle 1 HZP flux map with different asymmetric assembly modeling options.

\subsection{Cycle Operating Data}

Benchmark specification contains cycle operating data, core power level vs. calendar days (one average core power value per day for cycle 1 and power snap-shot values for cycle 2) that generates too many depletion points (500-600 points). Furthermore, the operating data is missing the control rod bank position, which is particularly essential during cycle start-up where core is not at full power and control rods moving. 
Accordingly, the cycle operation history has been condensed where the rod position can be extracted from available flux map data files. The condensed cycle 1 and cycle 2 depletion decks contain 66 and 45 state points, respectively. Additionally, the calendar days are converted to EFPD (Effective Full-Power Day) with the longest depletion step typically equal to 15 EFPD. Fig. 5 shows the real and condensed operating history for both cycles. The condensed history preserves the accuracy when performing the flux map comparison by averaging out the operating details between flux maps.
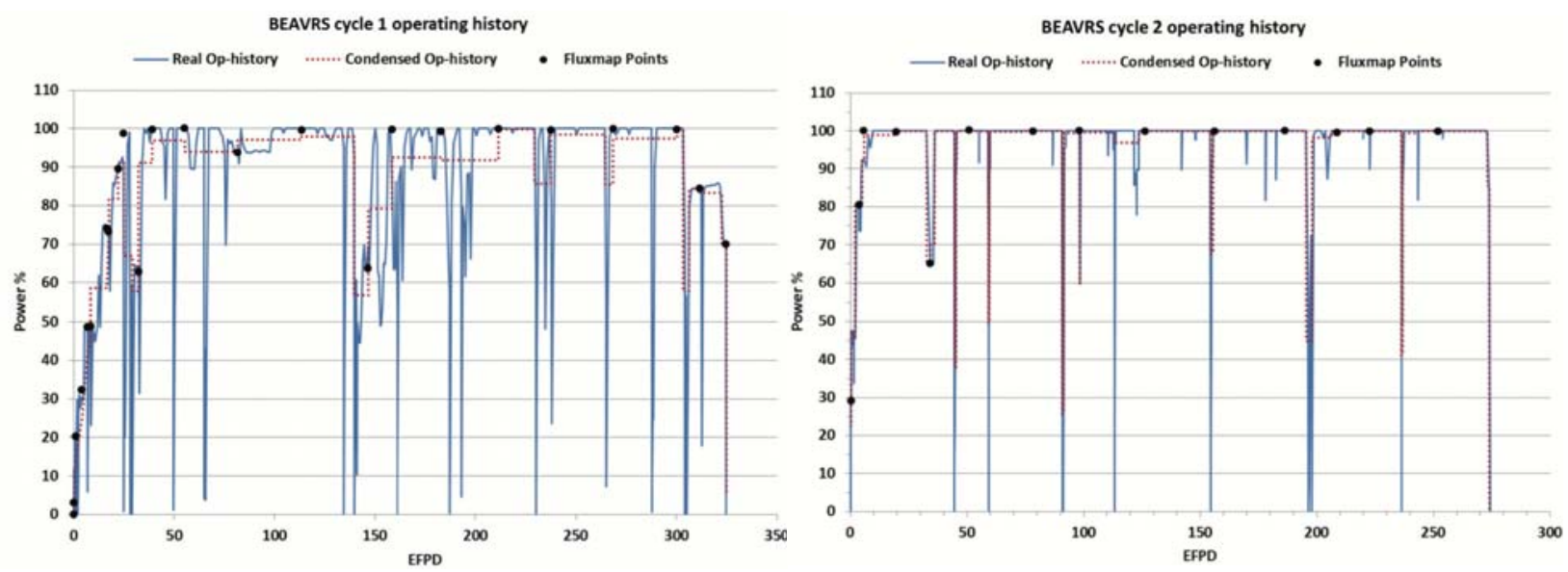

Figure 5. Cycle 1 and 2 power history vs EFPD.

\section{BENCHMARK RESULTS}

\subsection{Zero-Power Physics Testing}

The BEAVRS benchmark provides various zero-power physics test measurements: critical boron concentration, control rod bank worth, reactivity coefficients for cycle 1 and 2 , and also one flux map taken in cycle 1 at $0.71 \%$ power, which is discussed in section 4.3 . SIMULATE5 accuracy is presented in Table II.

Table II. Critical Boron Concentration at Zero Power.

\begin{tabular}{|l|r|r|r|r|}
\hline \multirow{2}{*}{ Rod Position } & Meas. & \multicolumn{3}{|c|}{ CMS5 - Measured } \\
\cline { 3 - 6 } & $(\mathbf{p p m})$ & SMPL/E7 & DFLT/E7 & DFLT/E8 \\
\hline ARO & 975 & -16 & -10 & 4 \\
\hline D In & 902 & -4 & 4 & 18 \\
\hline C, D In & 810 & -6 & 1 & 14 \\
\hline A,B,C,D In & 686 & -14 & -7 & -6 \\
\hline A,B,C,D,SE,SD,SC In & 508 & -20 & -15 & -6 \\
\hline \multicolumn{5}{|c|}{ Cycle 1 } \\
\hline ARO & 1405 & 10 & -9 & -10 \\
\hline C In & 1273 & 46 & 26 & 25 \\
\hline
\end{tabular}

The CMS5 critical boron concentration predictions for all rod configurations are in good agreement with the measurements. In Cycle 1 startup for all rods out (ARO) position, the default CMS5 model predictions with E7R1 library are within $10 \mathrm{ppm}$ against the measurement. The simplified model boron concentration is 5-8 ppm lower. The E8R0 library gives slightly higher, 5-15 ppm, critical boron concentration compared to E7R1 library. The differences between the simplified and default S5 models get larger with Cycle 2 
predictions. The maximum error is $46 \mathrm{ppm}$ overprediction for Bank-C inserted case with the simplified model.

Table III shows the comparisons between SIMULATE5 control rod bank worth predictions and the measured values for a number of different bank configurations. The maximum error in Cycle 1 is approximately $8 \%$ encountered for the shutdown bank-SE. The E8R0 library results have slightly larger error compared to E7R1 library. Although the simplified model yields good, even slightly better predictions in Cycle-1, the error with predicting the rod worth in Cycle-2 exceeds $10 \%$ for multiple rods.

Table III. Control Rod Bank Worth (pcm)

\begin{tabular}{|c|c|c|c|c|}
\hline \multirow{2}{*}{$\begin{array}{l}\text { Rod } \\
\text { Position }\end{array}$} & \multirow{2}{*}{$\begin{array}{l}\text { Rod Worth } \\
\text { (pcm) }\end{array}$} & \multicolumn{3}{|c|}{ (1 - CMS5/Meas)\% } \\
\hline & & SMPL/E7 & DFLT/E7 & DFLT/E8 \\
\hline \multicolumn{5}{|c|}{ Cycle1 } \\
\hline $\mathrm{D}$ & 788 & 0.6 & -1.5 & -1.9 \\
\hline $\mathrm{C}$ & 1203 & -0.1 & 0.9 & 1.5 \\
\hline $\mathrm{B}$ & 1171 & 2.3 & 0.3 & -0.2 \\
\hline A & 548 & -0.9 & 3.2 & 6.0 \\
\hline $\mathrm{SE}$ & 461 & 0.2 & 5.9 & 8.5 \\
\hline SD & 772 & 0.8 & 0.6 & 0.5 \\
\hline $\mathrm{SC}$ & 1099 & -0.8 & -0.6 & -1.0 \\
\hline \multicolumn{5}{|c|}{ Cycle 2} \\
\hline $\mathrm{D}$ & 426 & 6.4 & 5.3 & 5.3 \\
\hline $\mathrm{C}$ & 1014 & -3.9 & -1.8 & -2.2 \\
\hline B & 716 & -0.6 & -7.0 & -5.4 \\
\hline A & 420 & -9.1 & -2.4 & -4.5 \\
\hline SE & 438 & -6.3 & -3.3 & -4.3 \\
\hline SD & 305 & 12.1 & 6.2 & 7.3 \\
\hline $\mathrm{SC}$ & 307 & 11.3 & 5.2 & 6.4 \\
\hline SB & 781 & 0.1 & -2.0 & -1.6 \\
\hline SA & 326 & 10.7 & 3.0 & 4.7 \\
\hline Total & 4733 & 1.0 & 5.3 & 5.3 \\
\hline
\end{tabular}

Isothermal temperature coefficient $\left(\mathrm{pcm} /{ }^{\circ} \mathrm{F}\right)$ predictions are given in Table IV. In general, the ITC predictions in both cycles are in good agreement vs the measurement. The simplified S5 model resulted $0.2-0.3 \%$ larger error with the ITC predictions.

Table IV. Isothermal Temperature Coefficient $\left(\mathrm{pcm} /{ }^{\circ} \mathrm{F}\right)$

\begin{tabular}{|l|r|r|r|r|}
\hline \multirow{5}{*}{$\begin{array}{l}\text { Rod } \\
\text { Position }\end{array}$} & $\begin{array}{l}\text { ITC } \\
\mathbf{p c m} /{ }^{\circ} \mathbf{F}\end{array}$ & \multicolumn{4}{|c|}{ CMS5-Measured } \\
\hline \multicolumn{5}{|c|}{ Cycle 1 } \\
\hline ARO & -1.75 & -0.73 & -0.43 & 0.01 \\
\hline D In & -2.75 & -0.95 & -0.63 & -0.17 \\
\hline C, D In & -8.01 & -0.39 & -0.03 & 0.44 \\
\hline \multicolumn{5}{|c|}{ Cycle 2 } \\
\hline ARO & 1.71 & -0.02 & 0.20 & 0.30 \\
\hline
\end{tabular}

\subsection{Hot Full Power (HFP) Boron Predictions}

SIMULATE5 core follow calculations are performed using the condensed operating history as shown in Fig. 5. Fig. 6 presents the measured and CMS5 predicted critical boron concentrations. The CMS5 
predictions with different models/libraries are indistinguishable in these plots. The spikes with the predicted boron data in cycle 2 correspond to mid cycle outages for which the measurement is not available.
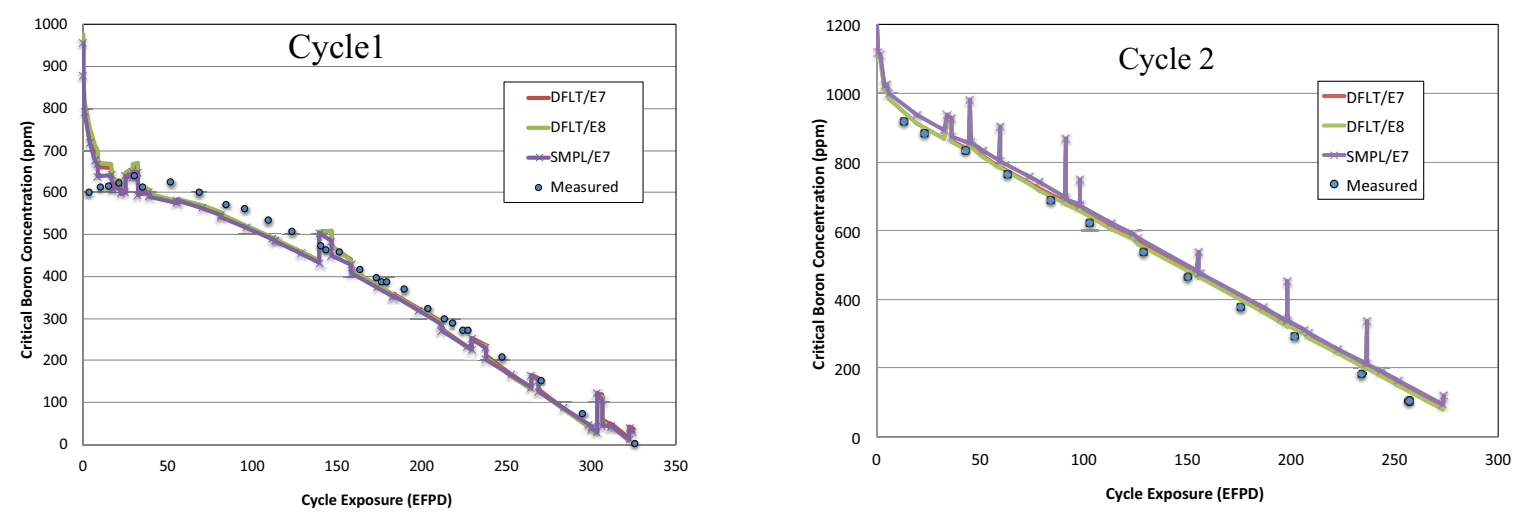

Figure 6. Cycle 1 and 2 core follow boron.

Except for the initial 150 EFPD of Cycle 1, the CMS5 reactivity predictions are in good agreement vs the plant measurement. There is a notable difference (Calc - Meas.) of around -35 ppm initially in the cycle (between 25 and 125 EFPD). This coincides with a period of operation rarely at full power. One possible explanation for the difference is that the measured boron concertation was adjusted to nominal $100 \%$ power and all-rods-out (ARO) condition, as this is a common practice in some utilities. To test the idea, a new depletion calculation at nominal condition is performed. Fig. 7 compares the predicted boron concentration with the actual vs nominal power history models against the measurement. Although the nominal depletion predictions at $\mathrm{BOC}$ is now closer to the measurement, the large deviations are still visible between $50 \mathrm{EFPD}$ to 150 EFPD and cannot be explained at this time.

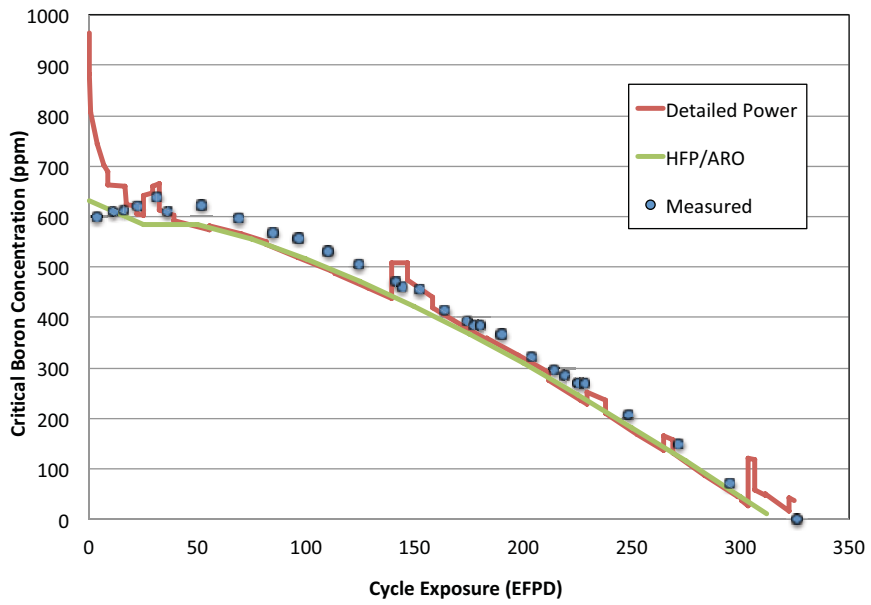

Figure 7. Cycle 1 core follow boron, detailed vs nominal (HFP/ARO) power depletion.

In the exposure interval from 50-300 EFPD, comparing calculated boron with CMS5 the DFLT/E7 model versus the measured boron gives an average bias of $-20 \mathrm{ppm}$. At the end of the cycle, the agreement is good with an average difference of $-16 \mathrm{ppm}$. In Cycle 2, the average bias between the CMS5 model prediction and the measurement is $22 \mathrm{ppm}$. 
The SMPL/E7 model predicts 15 to $25 \mathrm{ppm}$ lower critical boron concentration relative to DFLT/E7 model for the first 50 EFPD of cycle 1, where the cycle is rarely is at $100 \%$ power condition. It is believed that this difference is due the depletion models, where the microscopic depletion model, used with the default model, better captures the isotopic depletion/build-up at non-nominal conditions compared to the macroscopic depletion model, used with the simplified model. Over the entire cycle, the simplified model is less reactive by $5 \mathrm{ppm}$ in Cycle 1 and more reactive by $10 \mathrm{ppm}$ in Cycle 2 compared to the default S5 model.

Concerning the impact of the library, the higher reactivity observed with the E8R0 library at Cycle 1 startup quickly burns out after the first 100 EFPD operation. Overall, compared to the E7R1 library, the E8R0 library results in 3 and 6 ppm lower boron in Cycles 1 and 2, respectively.

\subsection{Flux Map Comparisons}

The 2D-assembly, 3D-nodal and 1D-axial (one value for each axial plane) comparisons of the pre-processed measured and calculated detector reaction rates at the measured assembly locations are given in Table $\mathrm{V}$ for Cycles 1 and 2 with all three models. The Cycle 1 flux map taken at low power on Day 7 (at 4\% power), which exhibits unusually large error, as much as $60 \%$ in $3 \mathrm{D}$, is excluded from comparisons. The results presented include all flux maps taken at other low power as well as at hot full power conditions. Table $\mathrm{V}$ shows that most low power flux maps, taken at non-equilibrium xenon condition, results in larger error compared to flux map taken at full power. Table VI presents the flux map statistics for all flux maps and those taken above $90 \%$ power, separately.

The noted RMS values, combined for 23 flux maps with power $>90 \%$, show excellent predictions with the $2 \mathrm{D}$ radial below $1.6 \pm 0.9 \%$ and the $3 \mathrm{D}$ nodal below $3.8 \pm 0.8 \%$ for HFP conditions. The flux map statistics in general are insensitive (less than $0.2 \%$ difference) to the choice of the nuclear data library. The advanced CMS5 model, specifically for cycle 2, provided better detector response predictions compared to the simplified model.

\section{CONCLUSIONS}

The MIT BEAVRS benchmark is evaluated with Studsvik's CMS5 code package using nuclear data libraries (ENDF/B-VII.R1 and ENDF/B-VIII.R0) and two different combination of modeling options; one using all advanced, SIMULATE5's default, computational models and the other using so called simplified options, employed by most nodal codes. Additionally, the importance of correct modeling of the asymmetric fuel assemblies with the nodal codes is demonstrated. The default CMS5 model calculated core parameters at zero and full power are in good agreement with the plant measurements: The boron predictions are within $30 \mathrm{ppm}$ at zero power and less than $20 \mathrm{ppm}$ at hot full power condition; the control rod worth is predicted with $0.7 \pm 3.8 \%$ accuracy; the radial and nodal reaction rate distributions are predicted with a mean difference of about $1.6 \%$ and $3.8 \%$, respectively. The simplified CMS5 predictions are slightly less accurate compared to the default model, particularly for the HZP calculations and the reaction rate evaluations. No significant difference is observed in predicting measured plant parameters with different nuclear data libraries.

As a concluding remark, it is acknowledged that BEAVRS is very useful benchmark for code validation. However, the lack of detailed operating history, such as control rod bank position for cycle 1 core follow, as well as large un-explainable radial power tilt with initial cycle-1 flux maps limit the number of data points that can conclusively be used for evaluating the performance of a core-analysis code and its computational models. 
Table V. Flux map radial, axial, and nodal RMS.

\begin{tabular}{|c|c|c|c|c|c|c|c|c|c|c|}
\hline \multirow[b]{2}{*}{ Days } & \multirow{2}{*}{$\begin{array}{c}\% \\
\text { Power }\end{array}$} & \multicolumn{3}{|c|}{ Radial, 2D, RMS (\%) } & \multicolumn{3}{|c|}{ Axial, 1D, RMS (\%) } & \multicolumn{3}{|c|}{ Nodal, 3D, RMS (\%) } \\
\hline & & SMPL/E7 & DFLT/E7 & DFLT/E8 & SMPL/E7 & DFLT/E7 & DFLT/E8 & SMPL/E7 & DFLT/E7 & DFLT/E8 \\
\hline 0 & 1 & 4.9 & 4.8 & 5.1 & 3.0 & 3.1 & 3.1 & 6.4 & 6.2 & 6.5 \\
\hline 18 & 20 & 2.6 & 3.0 & 3.4 & 2.2 & 2.6 & 2.6 & 4.6 & 5.0 & 5.3 \\
\hline 54 & 32 & 2.3 & 2.7 & 3.1 & 2.2 & 2.6 & 2.8 & 3.6 & 4.2 & 4.5 \\
\hline 62 & 49 & 2.7 & 3.0 & 3.3 & 6.7 & 6.4 & 6.1 & 7.8 & 7.7 & 7.6 \\
\hline 66 & 49 & 2.0 & 2.4 & 2.7 & 2.0 & 2.2 & 2.4 & 3.3 & 3.6 & 4.0 \\
\hline 81 & 74 & 2.0 & 2.2 & 2.5 & 3.8 & 4.1 & 4.3 & 4.8 & 5.1 & 5.4 \\
\hline 82 & 73 & 2.1 & 2.3 & 2.6 & 6.6 & 7.2 & 7.5 & 7.8 & 8.5 & 8.7 \\
\hline 88 & 90 & 1.9 & 2.0 & 2.2 & 2.6 & 1.8 & 2.0 & 3.9 & 3.2 & 3.5 \\
\hline 92 & 99 & 3.1 & 3.1 & 3.3 & 4.1 & 1.9 & 1.8 & 6.2 & 4.8 & 4.9 \\
\hline 161 & 63 & 4.6 & 4.6 & 4.6 & 1.4 & 1.5 & 1.7 & 5.6 & 5.6 & 5.7 \\
\hline 169 & 100 & 1.8 & 1.8 & 2.0 & 2.8 & 1.7 & 1.7 & 3.9 & 3.1 & 3.2 \\
\hline 187 & 100 & 1.8 & 1.9 & 2.0 & 2.8 & 1.9 & 1.9 & 3.9 & 3.2 & 3.3 \\
\hline 218 & 94 & 1.6 & 1.8 & 1.9 & 2.9 & 2.6 & 2.5 & 3.8 & 3.6 & 3.6 \\
\hline 251 & 100 & 1.5 & 1.7 & 1.9 & 2.1 & 2.1 & 2.2 & 3.3 & 3.3 & 3.4 \\
\hline 323 & 64 & 2.1 & 2.8 & 2.9 & 4.1 & 3.6 & 3.6 & 5.3 & 5.3 & 5.3 \\
\hline 339 & 100 & 1.5 & 1.7 & 1.9 & 2.0 & 2.4 & 2.4 & 3.4 & 3.8 & 3.9 \\
\hline 368 & 99 & 1.7 & 1.7 & 1.9 & 2.5 & 2.7 & 2.7 & 3.6 & 3.8 & 3.8 \\
\hline 403 & 100 & 1.8 & 1.9 & 2.0 & 2.9 & 2.9 & 2.9 & 3.9 & 4.0 & 4.0 \\
\hline 434 & 100 & 3.2 & 3.3 & 3.4 & 2.1 & 2.3 & 2.3 & 4.7 & 4.9 & 5.0 \\
\hline 468 & 100 & 1.6 & 1.7 & 1.9 & 1.7 & 1.7 & 1.8 & 3.2 & 3.3 & 3.4 \\
\hline 504 & 100 & 1.7 & 1.6 & 1.7 & 2.1 & 1.9 & 1.9 & 3.1 & 3.0 & 3.1 \\
\hline 551 & 85 & 2.0 & 2.0 & 2.1 & 2.1 & 3.0 & 3.0 & 3.6 & 4.2 & 4.2 \\
\hline 573 & 70 & 2.6 & 2.6 & 2.7 & 2.0 & 1.4 & 1.4 & 4.8 & 4.5 & 4.6 \\
\hline 5 & 29 & 3.2 & 3.3 & 3.2 & 3.9 & 3.3 & 3.4 & 5.6 & 5.2 & 5.3 \\
\hline 10 & 81 & 2.3 & 2.0 & 2.1 & 1.9 & 2.2 & 2.3 & 3.7 & 3.8 & 3.9 \\
\hline 13 & 100 & 1.9 & 2.0 & 2.0 & 4.5 & 4.7 & 4.8 & 5.5 & 5.7 & 5.7 \\
\hline 35 & 100 & 1.8 & 1.5 & 1.5 & 3.5 & 3.3 & 3.3 & 4.4 & 4.1 & 4.1 \\
\hline 42 & 65 & 1.9 & 1.6 & 1.6 & 2.0 & 1.8 & 1.9 & 3.3 & 3.0 & 3.1 \\
\hline 65 & 100 & 1.4 & 1.2 & 1.2 & 3.6 & 3.7 & 3.7 & 4.5 & 4.5 & 4.5 \\
\hline 93 & 100 & 1.4 & 1.0 & 1.0 & 2.3 & 2.3 & 2.3 & 4.0 & 3.9 & 3.9 \\
\hline 126 & 100 & 1.5 & 1.1 & 1.1 & 2.5 & 3.0 & 3.0 & 4.0 & 4.3 & 4.3 \\
\hline 156 & 100 & 1.5 & 0.9 & 0.9 & 2.2 & 2.5 & 2.5 & 3.2 & 3.3 & 3.3 \\
\hline 191 & 100 & 1.5 & 1.1 & 1.0 & 2.2 & 2.0 & 2.0 & 3.0 & 2.7 & 2.7 \\
\hline 220 & 100 & 2.0 & 1.5 & 1.5 & 2.4 & 2.3 & 2.3 & 4.3 & 4.0 & 4.0 \\
\hline 251 & 100 & 1.6 & 0.8 & 0.8 & 2.5 & 3.2 & 3.2 & 3.8 & 4.0 & 4.0 \\
\hline 266 & 100 & 1.6 & 1.0 & 1.0 & 2.2 & 2.1 & 2.1 & 3.1 & 2.8 & 2.8 \\
\hline 296 & 100 & 1.6 & 1.0 & 0.9 & 2.8 & 3.0 & 3.0 & 4.5 & 4.4 & 4.4 \\
\hline
\end{tabular}


Table VI. Flux Map Statistical Summary

\begin{tabular}{|l|l|l|l|l|l|l|l|}
\hline Power & $\begin{array}{l}\text { \# of } \\
\text { Maps }\end{array}$ & Assembly RMS and StDev (\%) & \multicolumn{2}{l|}{ Nodal RMS and StDev (\%) } \\
\cline { 3 - 7 } & SMPL/E7 & DFLT/E7 & DFLT/E8 & SMPL/E7 & DFLT/E7 & DFLT/E8 \\
\hline \multicolumn{7}{|c|}{ Cycle 1 } \\
\hline All & 23 & $2.3 \pm 0.9$ & $2.5 \pm 0.9$ & $2.7 \pm 0.9$ & $4.5 \pm 1.4$ & $4.5 \pm 1.4$ & $4.6 \pm 1.4$ \\
\hline$>90 \%$ & 12 & $1.9 \pm 1.0$ & $2.0 \pm 0.9$ & $2.2 \pm 0.9$ & $3.9 \pm 1.0$ & $3.7 \pm 0.8$ & $3.7 \pm 0.9$ \\
\hline \multicolumn{7}{|c|}{ Cycle 2 } \\
\hline All & 14 & $1.8 \pm 0.5$ & $1.4 \pm 0.7$ & $1.4 \pm 0.7$ & $4.1 \pm 1.0$ & $4.0 \pm 0.9$ & $4.0 \pm 0.9$ \\
\hline$>90 \%$ & 11 & $1.6 \pm 0.2$ & $1.2 \pm 0.3$ & $1.2 \pm 0.3$ & $4.0 \pm 0.7$ & $4.0 \pm 0.8$ & $4.0 \pm 0.8$ \\
\hline
\end{tabular}

\section{ACKNOWLEDGMENT}

The author acknowledges the former Studsvik employee Magnus Kruners for his work constructing the initial BEAVRS core model with CMS5.

\section{REFERENCES}

1. J. Rhodes, K. Smith and D. Lee, "CASMO5 Development and Applications," Proc. in PHYSOR 2006, Vancouver (2006).

2. T. Bahadir and S.-Ö. Lindahl, "Studsvik's Next Generation Nodal Code SIMULATE5," Proc. in Advances in Nuclear Fuel Management IV, Hilton Head Island, South Carolina, USA (2009).

3. N. Horelik and B. Herman, "MIT Benchmark for Evaluation and Validation of Reactor Simulations (BEAVRS)", MIT Computational Reactor Physics Group, Rev. 2.0.2. (2018).

4. N. Horelik, B. Herman, B. Forget, And K. Smith, "Benchmark for Evaluation and Validation of Reactor Simulations (BEAVRS), v1.0.1." in "Proc. Int. Conf. Mathematics and Computational Methods Applied to Nuc. Sci. \& Eng., Sun Valley, Idaho (2013).

5. Min Ryu, Yeon Sang Jung, Hyun Ho Cho \& Han Gyu Joo (2015): Solution of the BEAVRS benchmark using the nTRACER direct whole core calculation code, Journal of Nuclear Science and Technology, DOI: 10.1080/00223131.2015.1038664.

6. B. Collins, et al., "Simulation of the BEAVRS Benchmark using VERA," Int. Conf. Mathematics and Computational Methods Applied to Nuc. Sci. \& Eng., Jeju, Korea (2017).

7. V. Bykov, et all. "Solution of The BEAVRS Benchmark Using CASMO5 /SIMULATE5 Code Sequence," Proc. in PHYSOR 2016, Sun valley, Idaho (2016).

8. D. Kerdraon, et al., "ANDROMEDE calculations on BEAVRS benchmark (GABv2 / COCAGNE)," ICAPP 2019, International Congress on Advances in Nuclear Power Plants, ICAPP, France (2019).

9. T. Bahadir, "Improved PWR Radial Reflector Modeling with SIMULATE5," Proc. in Advances in Nuclear Fuel Management V, Hilton Head Island, South Carolina, USA (2015)..

10. R. Ferrer and J. Rhodes, "Generation and Initial Validation of a New CASMO5 ENDF/B-VIII.0 Nuclear Data Library," PHYSOR 2020, Cambridge, UK (2020).

11. K.S. Smith, "Nodal Diffusion Methods: Understanding Numerous Unpublished Details," Proc. in PHYSOR 2016, Sun valley, Idaho (2016). 